Original article

\title{
Grape polyphenols concentrate demonstrates cardioprotection in terms of hypoxic myocardial injury
}

\author{
Igor V. Zadnipryany ${ }^{1}$, Tatiana P. Sataieva ${ }^{1}$, Olga S. Tretiakova ${ }^{1}$, Anatoly V. Kubyshkin ${ }^{1}$, Walery Zukow ${ }^{2}$ \\ ${ }^{1}$ V.I. Vernadsky Crimean Federal University, Simferopol, Russia \\ ${ }^{2}$ Kazimierz Wielki University, Bydgoszcz, Poland
}

Received 20 April 2017, Revised 16 June 2017, Accepted 19 June 2017

(C) 2017, Zadnipryany I.V., Sataieva T.P., Tretiakova O.S., Kubyshkin A.V., Zukow W.

(C) 2017, Russian Open Medical Journal

\begin{abstract}
Objective - Beneficial effects of natural grape polyphenols on human health have been known for a long time and still attract more and more interest of many researchers. The aim of research was to reveal cardioprotective properties of polyphenols contained in grape concentrate "Fenocor" in rats exposed experimental histotoxic hypoxia.

Material and Methods - The study was conducted on 21 adult male Wistar rats divided into 3 groups, 2 of which were administered CoCl 2 water solution in $60 \mathrm{mg} / \mathrm{kg}$ dose for 7 days. The control group consisted of 5 non-exposed animals. The rats of the second group ( $\mathrm{n}=8$ ) after the administration of cobalt chloride did not receive any treatment, the animals of the third group $(n=8)$ after administration of cobalt chloride were additionally given Fenocor - a grape polyphelols water solution at a dose of $2.5 \mathrm{ml} / \mathrm{kg}$ diluted in $0.05 \mathrm{ml}$ of water. Morphological study was performed using light and electron microscopy. During the experiment the following biochemical parameters such as contents of malondialdehyde, oxidative modification of proteins were estimated.

Results - The outcome of the cobalt toxic exposure on the heart of animals in experiments resulted in the development of severe cardiomyopathy which lead to the need for timely cardioprotection. Histological structure of myocardium observed in the second group of male rats after cobalt intoxication on the background of grape polyphenol concentrate generally reflected a tendency to minimize of the damage extent which was manifested in the form of normalization of cell structures and muscle fibers.

Conclusion - Administration of Fenocor has demonstrated its antioxidant and cytoprotective properties, promoted myocardial structure preservation in rats exposed to histotoxic hypoxia.
\end{abstract}

Keywords: grape polyphenols, myocardium, hypoxia, cobalt, rats, experiment

Cite as Zadnipryany IV, Sataieva TP, Tretiakova OS, Kubyshkin AV, Zukow W. Grape polyphenols concentrate demonstrates cardioprotection in terms of hypoxic myocardial injury. Russian Open Medical Journal 2017; 6: e0404.

Correspondence to Walery Zukow. Address: Kazimierz Wielki University, ul. J.K. Chodkiewicza 30, 85-064 Bydgoszcz, Poland. Tel.: +48 $523419100 ;+485234$ 19 108. E-mail: w.zukow@wp.pl.

\section{Introduction}

The research of cardiotoxicity and myocardium ischaemia/hypoxia survival remains in the present field of research interest because the heart is the target organ of injury for many chemical compounds both medically prescribed and environmental [1]. Hence, the knowledge of structural changes in myocardial cells and the myocardium as a whole could explain the pathophysiology of myocardial diseases and allow correcting general therapeutic approaches. In our research we paid attention to the tissue (histotoxic) hypoxia which develops due to the inability of cells to utilize oxygen as a result of inactivation of tissue respiration enzymes. Industrial exotoxicant cobalt chloride $\left(\mathrm{CoCl}_{2}\right)$ in high doses is able to bind more firmly to the heme part of hemoglobin than iron and imitates the state of histotoxic hypoxia, which allows it to be used for modeling hypoxic states in an experiment [2]. In addition, cobalt by penetrating into cellular structures directly leads to the activation of lipid peroxidation, which severely aggravates hypoxic states [3].
Heavy metal cobalt is known as causative agent of toxic cardiomyopathy [2]. The primary scientific reports concerning the possible toxic role of cobalt in the heart failure reported its incidence the regular beer-drinkers [2, 3]. In 1965 investigators in Omaha and Quebec observed an endemic cardiomyopathy in heavy beer drinkers due to cobalt used as a beer additive. All beer drinkers were affected by unspecific gastroenteric disorders leading to serious anorexia followed by lethal cardiocirculatory failure manifested with dyspnea, hypotension, tachycardia, cyanosis and cardiomegaly with reduced cardiac output [3].

An important role in the treatment and prevention of adverse effects of the activation of free radical processes in histotoxic hypoxia is given to the antioxidant therapy - the promising direction of which is the use of various natural and synthetic antioxidants [4-6]. Despite the widespread use of antioxidants applied to protect the body from the damaging effects of lipid peroxidation stress, their scientifically proven effect on the antioxidant-prooxidant status of the organism exposed to histotoxic stress has not been adequately studied. 
In connection with the foregoing it is advisable to study the effect of various substances that may have antioxidant properties on the development of a hypoxic stress response. Unfortunately, most synthesized antioxidant substances are xenobiotics and, therefore, can themselves activate the processes of free radicals formation. Therefore our attention is drawn first of all to remedies of natural, in particular, vegetable origin $[6,7]$. Phenolic compounds are one of the most common secondary metabolites of higher plants. Their functions are extremely diverse and associated with the processes of photosynthesis, respiration, protection and resistance to many stresses. They have an important role in the system of antioxidant protection of plant cells, and this aspect has attracted much attention of researchers in recent years [6-8].

Although grapes contain a large number of polyphenols, the bioavailability of native polyphenols is extremely low. Polyphenols of berries are absorbed by the human body while eating grapes only in small amounts [8]. Consequently, as components of therapeutic polyphenols can be used in the composition of grape wines and food concentrates where they are present in bioavailable form. This requires further experimental and randomized clinical trials are required that assess the effect of the use of red wine and other polyphenolic products of grapes processing $[9,10]$.

Due to scientific progress our particular interest is drawn to the effects of new representatives of water-soluble grape food concentrates based on Enoant [11] technology and in particular we have interest in relatively new polyphenolic concentrate named "Fenocor" which contains the entire spectrum of polyphenols of grapes that exist in red wine but at the same time does not contain any alcohol. Mass concentration of phenolic substances in Fenocor is $82.69 \mathrm{~g} / \mathrm{dm}^{3}$ in comparison to Enoant with only $18.51 \mathrm{~g} / \mathrm{dm}^{3}$. Pioneer studies on animal models have revealed promising results of Fenocor particulary in treatment of cardiometabolic syndrome [12-14].

Therefore to continue cardiovascular research of grape polyphenols the objective of this study was to reveal the cardioprotective properties of grape food polyphenolic concentrate Fenocor in the experimental animals exposed to histotoxic hypoxic myocardial injury.

\section{Material and Methods}

\section{Study design}

The research was carried out on 21 mature male Wistar rats aged 10-12 months, weighing 150-210 g, which were divided into 3 experimental series. The first series (Control) included 5 intact animals. The second $(n=8)$ and third series $(n=8)$ rats were injected intravenously through the probe with an aqueous solution of $\mathrm{CoCl}_{2}$ at a medium toxic dose of $60 \mathrm{mg} / \mathrm{kg}$ dissolved in $1.5 \mathrm{ml}$ of distilled water once a day for 7 days [15]. In addition the animals of the third series after exposure to cobalt chloride afterwards received orally Fenocor polyphenol solution at a dose of $2.5 \mathrm{ml} / \mathrm{kg}$ mixed with $0.5 \mathrm{ml}$ of tap water via gastric tube for the antioxidant supplementation [14]. Fenocor was purchased from Magarach State Institution, Yalta.

Animals were housed in the University vivarium under conventional conditions at $19-22{ }^{\circ} \mathrm{C}$, with a $12 \mathrm{~h} / 12 \mathrm{~h}$ light/dark cycle. They had unlimited access to drinking water and food. Water and food intake was registered daily and no differences were observed between groups. The rats were sacrificed under chloroform anesthesia in accordance with the International Recommendations for Biomedical Research using Animals (1985) and the Rules of laboratory practice in the Russian Federation (Protocol No. 267 of the Ministry of Health of the Russian Federation of June 19, 2003). The experiments were carried out according to the permission of the Academic Council of the Crimean Medical Institute (Act No. 103 of 30.11.77). The animal maintenance was approved by the Institutional Committee on Bioethics and is consistent with the Guidelines for the Care and Use of Laboratory Animals published by the US NIH (No. 85-23, revised 1985).

\section{Polyphenolic composition of Fenocor}

The amount of total polyphenolic agents in the Fenocor concentrate is up to $82.69 \mathrm{~g} / \mathrm{dm}^{3}$, while in red wine it makes only $0.2-0.5 \mathrm{~g} / \mathrm{dm}^{3}$. According to phytochemical investigations oxygenoic acids and flavonoid polyphenols in the Fenocor composition are predominantly represented gallic acid, catechol, epicatechin, quercetin and procyanidins represented in Table 1 $[10,14]$.

As it can be seen from the data in Table 1 with an increase in the amount of phenolic substances in the grape concentrate an increase in antioxidant activity is observed.

Table 1. Concentration of phenolic substances and antioxidant activity of concentrates of polyphenols from red grapes

\begin{tabular}{|c|c|c|}
\hline \multirow[t]{2}{*}{ Index } & \multicolumn{2}{|c|}{ Gp concentrates } \\
\hline & Enoant & Fenocor \\
\hline \multicolumn{3}{|l|}{ Oxygenoic acids, mg/dm } \\
\hline - Gallic acid & 341.1 & 1119.2 \\
\hline - Lilac Acid & 22.6 & - \\
\hline - Amount & 363.8 & 1119.2 \\
\hline \multicolumn{3}{|l|}{ Flavan-3-ol, mg/dm } \\
\hline - (+) - 0-Catechol & 177.6 & 1752.6 \\
\hline - (-) - Epicatechin & 118.4 & 1374.2 \\
\hline - Amount . . . . . & 296.0 & 3126.8 \\
\hline \multicolumn{3}{|l|}{ Oxycinnamic acids, $\mathrm{mg} / \mathrm{dm}$} \\
\hline - Kaftaric acid & 11.7 & - \\
\hline - Cautaric acid & 1.8 & - \\
\hline - Amount & 13.5 & - \\
\hline \multicolumn{3}{|l|}{ Flavones, $\mathrm{mg} / \mathrm{dm}$} \\
\hline - Quercetin-3-O-glycoside & 3.1 & 15.4 \\
\hline - Quercetin & 49.6 & 10.2 \\
\hline - Amount & 52.7 & 25.6 \\
\hline \multicolumn{3}{|l|}{ Anthocyanins, $\mathrm{mg} / \mathrm{dm}$} \\
\hline - Petunidin-3-O-glycoside & 1.1 & - \\
\hline - Peonidin-3-O-glycoside & 0.6 & - \\
\hline - Malvidin-3-O-glycoside & 11.9 & - \\
\hline - Delphinidin-3-O-(6'-acetyl-glycoside) & 1.0 & - \\
\hline - Malvidin-3-O-(6'-acetyl-glycoside) & 1.8 & - \\
\hline - Petunidin-3-0-(6'-p-coumaroyl-glycoside) & - & - \\
\hline - Malvidin-3-O-(6'-p-coumaroyl-glycoside) & 2.5 & - \\
\hline - Amount & 18.9 & - \\
\hline \multicolumn{3}{|l|}{ Procyanidins and condensation products, $\mathrm{mg} / \mathrm{dm}^{3}$} \\
\hline - Oligomeric procyanidins & 603 & 4598 \\
\hline - Polymeric procyanidins & 28155 & 172662 \\
\hline $\begin{array}{l}\text { Antioxidant activity, } \mathrm{g} / \mathrm{dm}^{3} \text {, converted to the } \\
\text { standard Trolox units }\end{array}$ & 24.72 & 196.22 \\
\hline $\begin{array}{l}\text { Mass concentration of phenolic substances in } \\
\text { equivalence to the gallic acid, } \mathrm{g} / \mathrm{dm}^{3}\end{array}$ & 18.51 & 82.69 \\
\hline
\end{tabular}




\section{Light microscopy}

The extracted hearts were stopped in the diastole by immersing the organs in a $0.9 \%$ solution of $\mathrm{KCl}$. For the light microscopy paraffin sections 5-7 microns thick were made with a rotary microtome and stained with hematoxylin and eosin and Masson's trichrome for the detection of connective tissue elements according to a standard procedure [16]. The resulting microslides were examined using an Olympus CX-31 microscope (Japan). Morphometric measurements were made at 400x magnification by the licensed program Image J.

\section{Electron microscopy study}

For electron microscopy we took samples of left ventricular myocardium. Myocardium was prefixed for 2 hours in a $2.5 \%$ solution of glutaraldehyde in $0.1 \mathrm{M}$ phosphate buffer $\mathrm{pH}=7.2-7.4$ and then fixed in a $1 \%$ solution of osmium tetroxide on the same phosphate buffer at a temperature of $0-4^{\circ} \mathrm{C}$ for 1 hour. To ensure the targets for electron microscopic analysis the serial semi-thin sections 1-2 $\mu \mathrm{m}$ thick were made from all blocks stained with $1 \%$ methylene blue solution [17]. After identifying the necessary objects, the blocks were sharpened and ultrathin sections were made using the ultramicrotome of UMTP-7. After contrasting in a $2.5 \%$ solution of uranyl acetate and a $0.3 \%$ solution of lead citrate by E.S. Reynolds the microscopy of preparations was carried out on Selmi-125 electron microscope with an accelerating voltage of $125 \mathrm{kV}$.

\section{Immunohistochemistry research}

The immunohistochemistry (IHC) was performed on $3 \mu \mathrm{m}$ thick sections from $10 \%$ formalin fixed paraffin embedded tissues, according to the indirect tristadial Avidin-Biotin-Complex method of Hsu SM et al. We applied Anti-desmin Dako antibody Clone D33, diluted in ratio 1:50. Briefly, the procedure was: deparaffinization in xylene and alcohol series, rehydration, washing in phosphate saline buffer (PBS), incubation with normal serum, for 20 minutes, incubation with primary antibody overnight, standard labeled streptavidine-antibody biotin (LSAB) kit (DAKO), washing in carbonate buffer and development in 3,3'-DAB hydrochloride $/ \mathrm{H}_{2} \mathrm{O}_{2}$; microwave antigen retrieval in $\mathrm{M}$-citrate buffer pH 6.0 was performed. All specimens were counterstained with Meyer's Hematoxylin, examined and photographed on Olympus CX-31 microscope (Japan). To ensure the reliability of the experimental study, internal quality control of immunohistochemical techniques was performed as a part of an implemented and certified quality assurance system (ISO 9001/2001).

\section{Biochemical assays}

Biochemical studies of blood serum were performed in all groups of animals. During the experiments the following biochemical parameters were studied:

1. The content of malonic dialdehyde (MDA) which is as a secondary product of lipid peroxidation (LPO) was determined using diagnostic sets of TBC-Agate (Russia). The method for determining secondary products of lipid peroxidation is based on the reaction of MDA with thiobarbituric acid (TBA). The absorbance was measured at 535 and $570 \mathrm{~nm}$.
Calculation of TBA-active products was carried out according to the formula:

$$
C=\frac{D 535-D 570}{0,156} x 16,
$$

where $C$ is the optical density; D535 - wavelength equal to 535 $\mathrm{nm}$; D570 is a wavelength equal to $570 \mathrm{~nm}$. The content of TBAactive products was expressed in $\mu \mathrm{mol} / \mathrm{L}$.

2. In order to study the processes of oxidative modification of membrane proteins (OMP), we conducted studies to determine the carbonyl compounds formed during the modification of proteins in a state of oxidative stress caused by exposure to sodium nitrite. The study of spontaneous oxidative modification of proteins (OMP) was carried out by the method of Mihara and Levine in the modification of Dubinina [18]. Spectrophotometry was carried out at wavelengths of 270,274 and $363 \mathrm{~nm}$. The measurement results are presented in units of optical density. The study of biochemical indicators was carried out on a spectrophotometer SF-2000.

\section{Statistical analysis}

Statistical processing of data was carried out using licensed software Microsoft Office Excel 2007 and Statistica 10.0. Distribution of the morphometrical measurements was close to normal. As the variation and statistical parameters, the following parameters were calculated: minimum value $(\mathrm{min})$, maximum value (max), arithmetic mean (M), error of arithmetic mean $(m)$, standard deviation (SD), ratio of variation (Cv). Significance of differences in these groups was assessed by Student's criterion. A value of $p<0.05$ was considered significant.

The nonparametric quantitative data of the biochemical study due to small number of samples were presented as median (Me) and interquartile range $(L Q, U Q)$.

\section{Results}

\section{Myocardium changes during cobalt cardiomyopathy}

The data obtained during the study indicate that cobalt chloride $\left(\mathrm{CoCl}_{2}\right)$ possess a pronounced pathological effect on the myocardium of sexually mature male rats of the second group. Thus, general histology assessment with hematoxylin and eosin showed signs of hydropic dystrophy, microvascular dysfunction and lymphocytic infiltrations around lesioned cardiomyocytes (Figure 1a). The frequency and degree of lesions in different parts of the myocardium showed different pattern of distribution. Most commonly they were found in the left ventricle and interventricular septum, less often - in the wall of the right ventricle, even more rarely they were detected in the atria. Such damage mainly had the character of small-focal, often scattered and multiple. The Masson's trichrome staining revealed an excessive amount of collagen fibers in the interstitium, which apparently violated the transport of substrates and oxygen from the bloodstream to the working cardiomyocytes exacerbating already present hypoxic conditions (Figure 1b). In addition to that we observed signs of diffuse hemodynamic disorders, uneven capillary and venous plethora, desolation and spasm of numerous arterioles and capillaries accompanied by vivid perivascular edema. 
Table 2. Morphometric parameters of the myocardium of male rats, $M \pm S D$

\begin{tabular}{|c|c|c|c|c|}
\hline № & Index & I series: Control $(n=5)$ & II series: $\mathrm{CoCl}_{2}(n=8)$ & III series: $\mathrm{CoCl}_{2}+$ Fenocor $(n=8)$ \\
\hline 1. & Length of cardiomyocytes, $\mu \mathrm{m}$ & $73.10 \pm 3.87$ & $60.54 \pm 2.82^{*}$ & $65.12 \pm 3.14^{*}$ \\
\hline 2. & The area of cardiomyocytes, $\mu \mathrm{m}^{2}$ & $618.31 \pm 40.33$ & $397.29 \pm 20.88^{*}$ & $506.55 \pm 7.12^{*}+$ \\
\hline 3. & The area of the cytoplasm of cardiomyocytes, $\mu \mathrm{m}^{2}$ & $591.41 \pm 42.42$ & $356.47 \pm 27.69 *$ & $475.12 \pm 16.23 *+$ \\
\hline 5. & The diameter of the nuclei of cardiomyocytes, $\mu \mathrm{m}$ & $5.32 \pm 0.41$ & $5.01 \pm 0.26$ & $5.12 \pm 0.19$ \\
\hline 6. & The area of the nuclei of cardiomyocytes, $\mu \mathrm{m}^{2}$ & $24.71 \pm 2.19$ & $26.4 \pm 3.05$ & $25.58 \pm 0.08$ \\
\hline
\end{tabular}

$*-p<0.05$ compared to control animals series; $+-p<0.05$ compared to the animals of the second series without correction.
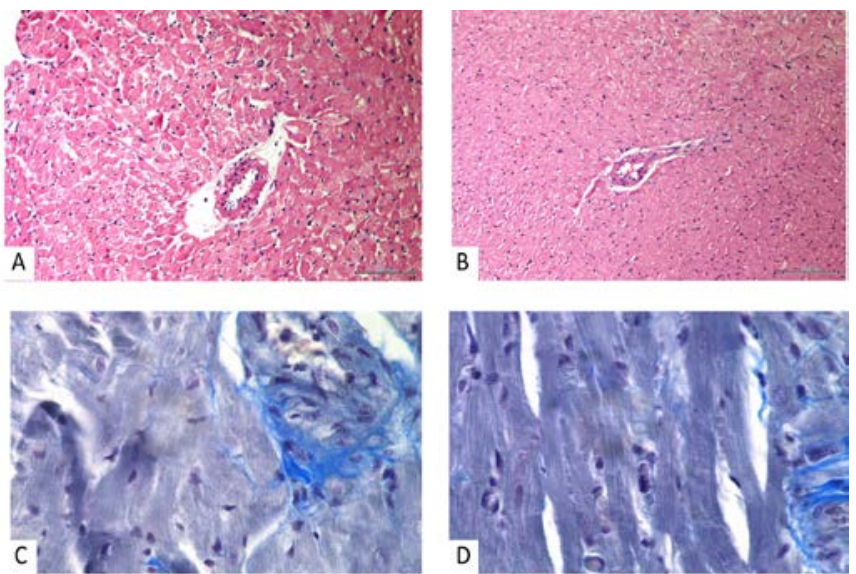

Figure 1. Light micrographs of myocardium tissue. Morphological changes in the myocardium of male rats exposed to histotoxic hypoxia. A) Exposure without treatment. Signs of myocardial edema, hydropic dystrophy, the presence of diapedic hemorrhages and the phenomena of sludge. Staining with hematoxylin-eosin. Mag. x200. B) Hypertrophic cardiomyocytes with signs of myocardial edema. Staining with hematoxylin-eosin. Mag. x200. C) Exposure without treatment. Increased amount of collagen fibers in the perivascular space. Masson's trichrome stain. Mag. x400. D) Moderate amount of collagen fibers in the perivascular space. Masson's trichrome stain. Mag. x400.
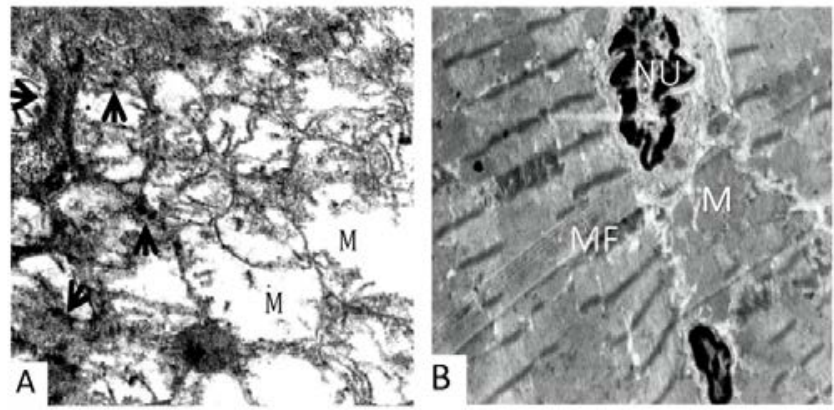

Figure 2. Ultrastructural changes in the myocardium of male rats exposed to histotoxic hypoxia (electron microscopy). A) Swelling of the sarcoplasm, subtotal destruction (arrowheads) of the mitochondrial cristae (M). TEM x19,000. B) Enlightenment of the nucleoplasm of the invaginated nucleus (NU), preservation of the structure of myofibrils (MF) and mitochondria (M) after polyphenolic correction. TEM x15,000.

Transmission-electron-microscopy (TEM) highlighted ultrastructural changes in the left ventricle of the rats exposed to cobalt chloride. Images show significant degenerative and subnecrotic changes in the myocardium in the II series of exposed rats. It was noted pronounced sarcoplasmic edema, lesions and ruptures in myofibril contraction bands. Ultrastructural study also revealed that exposure to cobalt chloride provoked significant mitochondrial suffering in the left ventricle which resulted in the subtotal loss of mitochondrial cristae, disruption of the mitochondrial membrane and finally their vacuolization with appearance dense osmophilic intramitochondrial particles (Figure 2a).

Finally, TEM images revealed that exposure to cobalt caused modification of the normal structure of the intercalated disks and dilation of some intercellular spaces with an abundant deposit of collagen fibers. Mitochondria represented cristae damage of different degrees, as well as partially or completely destroyed cellular organelles have been noted. The observed sites of sarcolemma damage caused the development of intracellular edema followed by destruction and other organelles. Similar pathological changes were also observed in endotheliocytes. Significant damage to their cell membranes was probably caused by the development of interstitial edema (Figure 2a). Increased cardiac collagen deposition can play an important role in the deteriorating function of the diseased heart [2].

Staining with hematoxylin-eosin followed by morphometry in the second experimental group without antioxidant treatment revealed a significant decrease in the length and area of cardiomyocytes, respectively, by $20.74 \%$ and $55.63 \%(p<0.05)$ that indicates their hypoxic, and in some cases, ischemic damage (Table 2).

To assess changes in the contractile apparatus of myocardial cells we used the anti-desmin antibody, knowing that desmin is the main intermediate filament protein that interacts with other proteins to form a continuous cytoskeletal network, which preserves the spatial relationship of the myocardiocytic contractile apparatus. Thus, desmin ensures cellular integrity, transport and mechanical and chemical signaling within cardiomyocytes. The evaluation of the immunohistochemical reaction to desmin allowed us to note that cobalt cardiomyopathy is characterized by a heterogeneous reaction, which means that myocardial cells contain varying amounts of desmin. In areas with an early interstitial collagen fibrosis and cell necrosis the reaction to desmin was reduced, which denotes some drastic reduction in cytoskeletal intermediate filaments and destruction of the contractile apparatus. It is possible that interstitial fibrillogenesis processes begin because of a chronic myocardial ischemia, which would explain cardiomyocyte suffering manifested by a reduction of intermediate filaments, cytoplasmic organelles and myofibrils (Figure 3a).

Increasing evidence suggests that oxidative stress may play a crucial role in different types of cardiac diseases. The study of products of lipid peroxidation, in particular of MDA in blood serum revealed its increase in histotoxic hypoxia by 3.64 times above normal (Table 3), and the level of carbonyl compounds exceeded the control parameters by 3.17 times, that indicates the rapid increase of peroxide oxidation of membrane lipids and oxidative modification of sarcolemmal proteins induced by increased formation of hydroxyl radicals leading to irreversible structural changes in cellular and intracellular membranes (Table 3). 
Table 3. Malonic dialdehyde (MD) and oxidative modification of membrane proteins (OMP) in the blood serum of intact and cobalt intoxicated rats, Me $(\mathrm{LQ}, \mathrm{UQ})$

\begin{tabular}{|c|c|c|c|c|}
\hline № & Index & I series: Control $(n=5)$ & II series: $\mathrm{CoCl}_{2}(n=8)$ & III series: $\mathrm{CoCl}_{2}+$ Fenocor $(n=8)$ \\
\hline 1 & $\mathrm{MDA}, \mu \mathrm{mol} / \mathrm{L}$ & $2.38(2.04,2.66)$ & $9.96(9.92,9.97)$ & $7.17(6.93,7.38)$ \\
\hline 2 & OMP, units & $0.16(0.15,0.17)$ & $0.62(0.61,0.64)$ & $0.51(0.47,0.53)$ \\
\hline
\end{tabular}

Values are expressed as median (Me) and interquartile range (LQ; UQ). Mann-Whitney U-test.

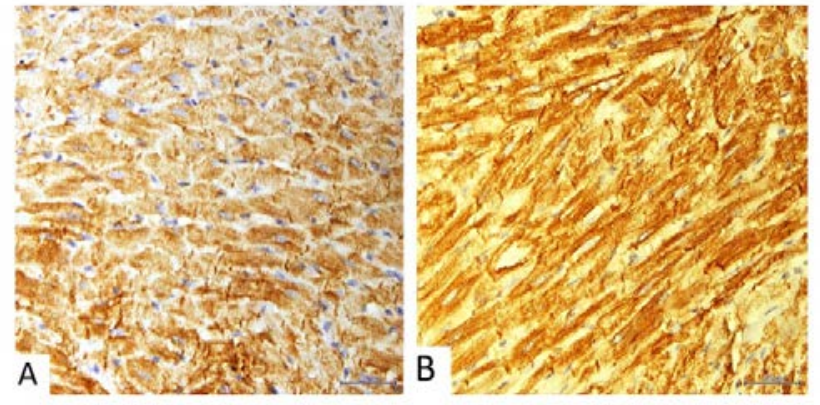

Figure 3. Immunohistochemical staining for desmin. A) Cross-section myocardiocytes with very variable immunohistochemical reaction to desmin, associated with areas with intracytoplasmic vacuolization, with no reaction to desmin. Mag. $\times \mathbf{4 0 0}$. B) Contractile cardiomyocytes show vivid expression of desmin. Mag. $\times 400$.

\section{Polyphenolic treatment of exposed animals}

The structure of the rat myocardium observed in the third series with Fenocor administration during cobalt intoxication generally reflected a tendency for minimization of the amount of myocardial hypoxic damage which was represented by intact nuclei of most cardiomyocytes, low amount of vacuoles and hemorrhages along with mostly preserved myofibrils (see Figure 1b). The vast majority of the myocardium structural components during histological examination looked moderately alterated. A decrease in the pathological permeability of the sarcolemma was noted, which was manifested by both diminished interstitial edema and dystrophy in most cardiomyocytes. However, there were present diffuse areas of myocardium showing dystrophy and necrosis, which was manifested by the homogenization of the cytoplasm or the appearance of significant enlightenment in it, which, as is known, is evidence of the development of dystrophic and prenectrotic processes [2]. The presence of hypertrophic cardiomyocytes and compensatory fusion of some of them were observed.

Morphometry estimated the length and area of cardiomyocytes decreased in comparison with the control by $13.28 \%$ and $18.07 \%$, respectively $(p<0.05)$, which exceeded the same parameters in the second experimental series without antioxidant protection by $7.46 \%$ and $33.56 \%$, which indicated the cytoprotective effect of Fenocor (see Table 2).

Masson's trichrome staining revealed thin layers of connective tissue in the interstitium, as well as in the perivascular space (see Figure 1d).

According to the results of electron microscopy, the cardiomyocytes of male rats after the 7-day of Fenocor administration were damaged matching irreversible necrotic and subnecrotic and predominantly reversible dystrophic changes.
Some cardiomyocytes presented quite normal morphological features.

Reversible changes were represented by uneven distribution of nuclear chromatin, distruction of Z-discs, the focal lysis of mitochondrial cristae but many mitochondria still retained their normal structure (Figure 2b). However, the cytoprotective effect of the above antioxidant still could not completely compensate for the destructive effect of powerful hypoxic stress. This was evidenced by discovery of cardiomyocytes showing vivid edema in the sarcoplasm where areas o of myofibril lysis with numerous vacuoles were noted. It should be noted that in such cells sometimes there were found quite large mitochondria, which had no significant structural damage. This apparently is also explained by the positive compensatory effect of antioxidant correction. The incomplete compensation of the effect of hypoxic stress was also evidenced by diffuse fibrogenesis in the interstitium of cardiomyocytes.

This result correlates with desmin content dynamics. Desmin is necessary to determine localization and regulate cell permeability. Ultrastructural analysis of different types of cells showed the obvious connection between mitochondria and desmin intermediate filaments [3]. Apart of ruined cells most of cardiomyocytes in the left ventricle expressed structural desmin fibers. Moreover, there was a compensatory increase of the relative content of desmin in some cardiomyocytes (Figure 3b).

According to the measured lipoperoxidation, protein carbonyl contents were also strongly decreased after polyphenolic supplementation. The combined decreased of both biomarkers of oxidative stress after supplementation is a strong indication of the efficiency of Fenocor to counteract the progressive oxidative insult related to cobalt hemic hypoxia. In the present study grape polyphenols attenuated the cobalt-induced oxidative stress. The antioxidant effect of the Fenocor concentrate was manifested in a significant decrease in the serum concentration of MDA products compared to the second test group without correction by $16.28 \%$ (see Table 3). Concentration of OMP products also decreased in comparison to the second series and exceeded the control figures by $67.30 \%$.

The study of cardiomyocytes in rats of the third series showed that myocardium has more moderately damaged and intact area. Though we cannot state that it can completely prevent the hypoxic impairment. But still polyphenols are able bind free radicals $[4,6]$ and make it possible to reduce the amount of free radicals, to obtain a greater amount of ATP even in conditions of insufficient substrates and to prevent the progression of oxidative stress in the early stages of ischemic and hypoxic damage to the myocardium, which was confirmed in this study.

\section{Discussion}

The possible mechanisms of cobalt toxicity on the rat myocardium can be assumed as follows: 
1. The acute cardiotoxicity of cobalt can be related to a competitive inhibition of $\mathrm{Ca}^{2+}$ that was reflected as the inability of cardiomyocytes to utilize high energy phosphate and to maintain mechanical tension in the state of excitation. In addition lipid peroxidation processes catalyzed by cobalt lead to irreversible lesions of cell membranes [2].

2. High concentrations of cobalt ions violate myocardial energy metabolism by inhibition of transaminases activity by affecting succinate dehydrogenase and $\alpha$-ketoglutarase. So pyruvic and lactic acids accumulate in the mitochondria and elevate osmotic pressure resulting in water binding, oedema and eventually lead to structural disruption that is manifested in mitochondrial disfunction $[19,20]$.

Thus, the result of the experimental influence of cobalt chloride on the myocardium resulted in the development of severe toxic cardiomyopathy, which requires antioxidant therapy.

One of the new cardioprotective promising natural remedies is the polyphenols of the Crimean grapes. They are not synthesized in the human body and enter into it only with plant food. The group of high-molecular polyphenols acts as active antioxidants, which can suppress chain free radical reactions, decrease the activity of oxidative enzymes and decrease the concentration of peroxide lipids in the blood plasma [21].

Nowadays during the backdrop of a wide range of modern pharmacotherapeutic agents and various methods of non-drug treatment, grapes and products of its processing do not only retain their value but also show important and unique antioxidant effects. One of the most striking and convincing manifestations is the world-famous "French paradox" - a much lower incidence of angina pectoris and other cardiovascular pathologies among residents of certain French regions who regularly and moderately consume red wines compared with the population of other developed countries. A reliable inverse correlation was also established between mortality from myocardial infarction and moderate consumption of red dry wine. These facts unequivocally testify the powerful therapeutic and preventive effects of anthocyanins of grapes like well-known quercetin and resveratol, which are part of the traditional French dry red wines [11, 21].

Although the berries of the Crimean grapes contain a large number of polyphenols, the bioavailability of native polyphenols is extremely low. Only in small amounts of native polyphenol berries are absorbed by the human body when eating grapes for food. As components of spa treatment technologies, polyphenols can be used in grape wines and food concentrates, where they are contained in bioavailable form [8]. Grape wines have not yet been fully studied from the point of view of their effectiveness in the treatment of cardiac pathology which is largely due to the negative effects of relatively large doses of alcohol included in their composition. The form of practical application of polyphenols of the Crimean grapes "Cabernet-Sauvignon" as a component of spa treatment in the health resorts of the Southern coast of Crimea is the "Enoant" non-alcoholic food concentrate developed at the Institute of Grape and Wine "Magarach", Russian Federation, where the concentration of total polyphenols reaches 18-20 g/l. Since 2000 the clinical research has begun to reveal this red grape polyphenolic concentrate health effects in the treatment of sanatoriums in patients suffering from cardiac and respiratory pathologies [11, 14].

According to the results of preliminary studies, it is known that the antioxidant activity of total combination of grape polyphenols in Enoant concentrate is more than 3000 times higher than the antioxidant activity of blood plasma [9].

Clinical research has shown a broad variety of therapeutic and preventive effects of the consumption of "Enoant". For instance, it has been proven that its liver and kidney protective activity allows to use it for the correction of toxic effects of anti-cancer therapy with citostatics; its antistreptococcus and enterococcus activity is effective during general paradontitis therapy; good results have been achieved during the rehabilitation of chronic and recurrent lung illnesses and other cardiac - respiratory system conditions.

The results obtained by us correspond with the data of other authors. Thus, in a study on 48 Wistar rats with a model of metabolic syndrome induced by fructose, pronounced antioxidant effects were achieved after administration of the food enriched in polyphenols [6]. In yet another experimental study involving metabolic syndrome the antihypertensive activity of oral administration of polyphenol extract from grape seeds was proven with a maximum effect at a dose of $375 \mathrm{mg} / \mathrm{kg}$ [12]. Along with the experimental data, polyphenols demonstrated efficacy (blood glucose decreased, insulin secretion and sensitivity increased) in some randomized clinical trials [13].

Antioxidant activity of polyphenolic concentrates occurs due to the presence of flavonoid (anthocyanins, quercetin, rutin, catechins, epicatechin, leucoanthocyanins, and tannins) and nonflavonoid (gallic and lilac acids, resveratrol, coffee and ellagic acids) components. The Enoant, in previous researches [10] and Fenocor concentrates used in this experiment have demonstrated pronounced antioxidant activity, as well as a positive effect on the endogenous antioxidant potential on myocardium. The greatest effect on the normalization of the oxidation-antioxidant imbalance was demonstrated by Fenocor. Treating animals with the grape polyphenols significantly attenuated the detrimental changes observed in the myocardium, but yet it failed in complete preventing cobalt-induced cardiac damage. These observations could be explained by the fact that $\mathrm{CoCl}_{2}$ exerts its toxic effects by several other mechanisms, besides affecting the cellular redox balance.

\section{Conclusion}

Heavy metal Cobalt is regarded as being responsible for a form of toxic cardiomyopathy which pathogenesis and clinical description must still find a specific place in the range of congestive cardiomyopathies. Histological studies revealed that experimental oral of cobalt chloride to the mature male rats causes a complex of reactive-dystrophic changes in the myocardium. This occurs due to the development of histotoxic hypoxia which is accelerating membrane-damaging lipid peroxidation processes.

The polyphenolic antioxidant activity of Fenocor concentrate due to suppression of lipid peroxidation and oxidative modification of proteins leads to the blockage of oxidative stress - an important pathogenetic mechanism of cellular membrane damage in histotoxic hypoxia. So our findings indicate that grape polyphenols intake may be a good nutritional strategy to reduce histotoxic cardiac damage.

\section{Ethics approval}

Animal experiment was approved by the Bioethics committee of Crimea Federal University Center (Protocol № 8 from 15.03.2016) according to the permission of the Academic Council of the Crimean 
Medical Institute (No. 103 of 30.11.77). The study was approved by the Institutional Committee on Bioethics and is consistent with the International Guidelines for the Care and Use of Laboratory Animals published by the US NIH (No. 85-23, 1985) and Guide for the Care and Use of Laboratory Animals (2009).

Conflict of interest: none declared.

\section{References}

1. Buja LM. The pathobiology of acute coronary syndromes: clinical implications and central role of the mitochondria. Tex Heart Inst J 2013; 40(3): 221-228. https://www.ncbi.nlm.nih.gov/pubmed/23914009.

2. Wiberg GS, Munro IC, Morrison AB. Effect of cobalt ions on myocardial metabolism. Can J Biochem 1967; 45: 1219-1223. https://doi.org/10.1139/o67-142.

3. Hatori N, Pehrsson SK, Clyne N, Hansson G, Hofman-Bang C, Marklund $\mathrm{SL}$, et al. Acute cobalt exposure and oxygen radical scavengers in the rat myocardium. Biochim Biophys Acta 1993; 1181: 257-260. https://doi.org/10.1016/0925-4439(93)90029-z.

4. Zadnipryanyi IV, Tretyakova OS, Sataeva TP. Investigation of the antioxidant activity and cardioprotective effect of reamberin and cytoflavin in newborn rats exposed to chronic hemic hypoxia. Arkhiv Patologii 2015; 77(6): 39-44. https://doi.org/10.17116/patol201577639-44.

5. Novikov VE, Levchenkova OS. Promising directions of search for antihypoxants and targets of their action. Eksperimentalnaya $i$ Klinicheskaya Farmakologiya 2013; 76(5): 37-47. Russian. https://elibrary.ru/item.asp?id=22392391.

6. Jaroslawska J, Wroblewska M, Juskiewicz J, Brzuzan L, Zdunczyk Z. Protective effects of polyphenol-rich blackcurrant preparation on biochemical and metabolicbiomarkers of rats fed a diet high in fructose. J Anim Physiol Anim Nutr (Berl) 2016; 100(1): 136-145. https://doi.org/10.1111/jpn.12321.

7. Santangelo C, Vari R, Scazzocchio D, Di Benedetto R, Filesi C, Masella R. Polyphenols, intracellular signalling and inflammation. Ann Ist Super Sanita 2007; 43(4): 394-405. https://www.ncbi.nlm.nih.gov/pubmed/18209273.

8. Tamura H, Yamagami A, Agric J. Antioxidative activity of monoxylated antocyanins isolated from Muscat bailey A grape. Food Chem 1994; 42: 1612-1615. https://doi.org/10.1021/jf00044a005.

9. Zajcev GP, Catrich LI, Ogai JuA. Biologically active polyphenols of the dry red 'cabernet sauvignon' wine material and the food concentrate 'EnoanT'. Magarach. Vinogradarstvo i Vinodelie 2010; (3): 25-27. Russian. https://elibrary.ru/item.asp?id=23577157.

10. Mizin VI, Yezhov VV, Severin NA, Kruglova AY. Syndrome pathogenic approach to application of therapeutic and prophylactic effects of grape polyphenol complex under pathology of cardiovascular system. Journal of Health Sciences 2013; 3(16): 95-108. Russian.

11. Meshkov VV, Bogdanov NN, Bogdanov AN. The experimental conditions to optimize the use of techniques "Enoant". Vestnik Fizioterapii i Kurortologii 2002; (2): 30-33. Russian.

12. Pons Z, Margalef M, Bravo FI, Arola-Arnal A, Muguerza B. Acute administration of single oral dose of grape seed polyphenols restores blood pressure in a rat model of metabolic syndrome: role of nitric oxide and prostacyclin. Eur J Nutr 2016; 55(2): 749-758. https://doi.org/10.1007/s00394-015-0895-0.

13. Bozzetto L, Annuzzi G, Pacini G, Costabile G, Vetrani C, Vitale M, et al. Polyphenol-rich diets improve glucose metabolism in people at high cardiometabolic risk: a controlled randomised intervention trial. Diabetologia 2015; 58(7): 1551-1560. https://doi.org/10.1007/s00125015-3592-x.

14. Avidzba AM, Kubyshkin AV, Guguchkina TI, Markosov VA, Katsev AM, Naumova NV, et al. The antioxidant activity of the products of processing of red grape of Cabernet Sauvignon, Merlot, Saperavi.
Voprosy Pitaniia 2016; 85(1): 99-109. Russian. https://elibrary.ru/item.asp?id=25596080.

15. Clyne N, Hofman-Bang C, Haga Y, Hatori N, Marklund SL, Pehrsson SK, et al. Chronic cobalt exposure affects antioxidants and ATP production in rat myocardium. Scand J Clin Lab Invest 2001; 61: 609-614. https://doi.org/10.1080/003655101753267964.

16. Merkulov GA. The course of pathological and histological tehniki. Moscow, Russia: Medicine, 1969. Russian.

17. White K. Electron Microscopy: Methods and Protocols (3rd Ed.). Kuo John (Ed.). Humana Press, Totowa, NJ, 2014,799 pages. ISBN: 978 1627037754. Microscopy and Microanalysis 2014; 20(5): 1624 https://doi.org/10.1017/s143192761401321x.

18. Mihara M, Uchiyama M. Determination of malonaldehyde precursor in tissues by thiobarbituric acid test. Anal Biochem 1978; 86(1): 271-278. https://doi.org/10.1016/0003-2697(78)90342-1.

19. Bruick RK. Oxygen sensing in the hypoxic response pathway: regulation of the hypoxia-inducible transcription factor. Genes Dev 2003; 17: 2614-2623. https://doi.org/10.1101/gad.1145503.

20. Mohiuddin SM, Taskar PK, Rheault M, Roy PE, Chenard J, Morin Y. Experimental cobalt cardiomyopathy. Am Heart J 1970; 80 (4): 532543. https://doi.org/10.1016/0002-8703(70)90202-4

21. Tsao R. Chemistry and biochemistry of dietary polyphenols. Nutrients 2010; 2(12): 1231-1246. https://doi.org/10.3390/nu2121231.

\section{Authors:}

Igor V. Zadnipryany - MD, Department of Topographical Anatomy, Medical Academy n.a. S.I. Georgievsky, V.I. Vernadsky Crimean Federal University, Simferopol, Russia. http://orcid.org/0000-0002-8181-9709.

Tatiana P. Sataieva - MD, Department of Medical Biology, Medical Academy n.a. S.I. Georgievsky, V.I. Vernadsky Crimean Federal University, Simferopol, Russia. http://orcid.org/0000-0001-6451-7285.

Olga S. Tretiakova - MD, Department of Social Medicine and Public Health Economics, Medical Academy n.a. S.I. Georgievsky, V.I. Vernadsky Crimean Federal University, Simferopol, Russia. http://orcid.org/0000-0002-24760624.

Anatoly V. Kubyshkin - MD, Professor, Head of General and Clinical Pathophysiology Department, V.I. Vernadsky Crimean Federal University, Simferopol, Russia. http://orcid.org/0000-0002-1309-4005.

Walery Zukow - MD, Faculty of Physical Education, Health and Tourism, Kazimierz Wielki University, Bydgoszcz, Poland. http://orcid.org/0000$\underline{0002-7675-6117}$ 